Cesar Winnicki ${ }^{a}$

Bernd Orzessek ${ }^{\mathrm{a}}$

\title{
Chronisch-entzündliche Darmerkrankungen aus der Sicht der Komplementärmedizin - Teil 1
}

Immer mehr und immer jüngere Menschen erkranken an chronischentzündlichen Darmerkrankungen (CED) - mit den zwei klassischen Vertretern Morbus Crohn und Colitis ulcerosa [1]. Obwohl in den letzten Jahren für die Behandlung von CED neue Medikamente wie z.B. TNF (Tumornekrosefaktor)-alpha-Blocker zugelassen wurden, gelingt es selten, die chronische Darmentzündung vollständig auszukurieren. In der Regel müssen die Betroffenen, trotz langfristiger medikamentöser Therapie, regelmässige Exazerbationen in Kauf nehmen. Aufgrund dieser unbefriedigenden Situation suchen viele CED-Patienten nach komplementären Behandlungsmethoden. Doch was können die ganzheitliche Betrachtung dieser komplexen Erkrankung und der entsprechende komplementäre Therapieansatz genau zum Management von CED beitragen?

\section{Darm - Grenzfläche zwischen Innen- und Aussenwelt}

Die Darmwand besitzt aufgrund ihrer speziellen anatomischen Struktur aus Falten, Zotten und Microvilli eine Oberfläche von über $400 \mathrm{~m}^{2}$. Das Ökosystem der Darmflora besteht neben Archaeen und Eukaryoten aus überwiegend anaeroben Bakterien im Dickdarm sowie fakultativ anaeroben Bakterien im Dünndarm. Es ergibt sich eine Gesamtzahl von 10-100 Billionen Bakterien - etwa

${ }^{a}$ Aeskulap-Klinik, Brunnen, Schweiz ebenso viele wie Zellen im Körper des Menschen. Mittlerweile sind durch molekulargenetische Analysen bis zu 36000 Arten bekannt, von denen sich jedoch nur ein Teil kultivieren lässt. Durch die sogenannte Kolonisationsresistenz wird die Grenzfläche vor pathologischen Erregern geschützt. Daneben besorgt die Darmflora wichtige Aufgaben in der Verdauung, der Biosynthese von Vitaminen und kurzkettigen Fettsäuren sowie der Immunmodulation [2].

Die humane Darmflora ist Teil des sogenannten Mikrobioms, ein von Joshua Lederberg (1925-2008) eingeführter Begriff, der die gesamten Mikroben des menschlichen Körpers, deren Genome und deren Interaktionen mit ihrer Umwelt umfasst [3]. Im Rahmen dieses Blickwinkels wird die Physiologie des Menschen nicht mehr isoliert als Individuum, sondern immer nur zusammen mit seinen assoziierten mikrobiellen Gemeinschaften betrachtet. Ein Beispiel hierfür mag die Entdeckung der in der Mukosa des Dünndarms gelegenen Th17-Zellen sein, die eine Funktion in der Abwehr von definierten intestinalen Bakterien und Pilzen wahrnehmen, aber je nach Differenzierung auch eine herausragende Rolle in der Genese von Autoimmunerkrankungen spielen. Diese entscheidende Differenzierung wird durch das Mikrobiom induziert [4].

Der aus dem Prinzip der Entsprechungen bekannte Lehrsatz «Wie im Kleinen, so im Grossen» wird anschaulich, wenn wir die Darmwand als Grenzfläche zwischen Innen- und Aussenwelt verstehen lernen. Die Notwendigkeit, zugleich zwei völlig gegensätzliche Funktionen zu erfül- len, zum einen als Resorptionsorgan und zum anderen als Barriere vor Noxen, Nahrungsmittelantigenen oder Pathogenen, stellt die Grenzfläche Darmschleimhaut vor eine anspruchsvolle Herausforderung. Die intestinale Barriere besteht aus drei Funktionsebenen: anatomisch, mikrobiologisch und immunologisch. Geht die immunogene Balance verloren, kommt es entweder zu überschiessenden Abwehrreaktionen oder zu einer vermehrten Durchlässigkeit der Darmwand, was den Körper bedingungslos der Aussenwelt ausliefert.

Diese Ambivalenz zwischen lebensnotwendigen Funktionen finden wir auch «im Grossen», in unserem sozialen Leben. Auch hier gilt es, in jedem Augenblick die Balance zu finden zwischen Abwehr, und damit einem Schutz vor Verletzung, und dem Wunsch nach Kontakt und Geborgenheit. Weder im Kleinen noch im Grossen können wir diese Aufgabe jederzeit perfekt ausfüllen. Wie bei vielen biologischen Prozessen kann hier nur die Schwingung zwischen zwei gegensätzlichen Polen eine funktionelle Stabilität gewährleisten. Hierfür ist eine ausreichende Regulationsfähigkeit notwendig.

Im Vorfeld des Versagens der Barrierefunktion der Darmschleimhaut (Leaky-Gut-Syndrom) kommt es zur Schwächung einer oder mehrerer Schutzfunktionen der Darmwand wie der Darmflora, der Schleimschicht oder des sekretorischen Immunglobulin (Ig) A. Dies kann wiederum durch medikamentöse Behandlungen, Infektionen, Stress, ungünstige Ernährung, Nahrungsmittelzusatz-

\section{KARGER}

Fax +49761 4520714 Information@Karger.com www.karger.com
(๑) 2013 S. Karger GmbH, Freiburg
Dr. med. Cesar Winnicki

Chefarzt Aeskulap-Klinik

Gersauerstrasse 8, 6440 Brunnen, Schweiz

Cesar.Winnicki@aeskulap.com 
stoffe und Nahrungsmittelallergien bzw. -intoleranzen ausgelöst werden [5]. Die Folge ist eine entzündliche Reaktion, die zu einem Versagen von «Tight Junctions» bzw. zu einem Absterben von Becherzellen führt. Auf mikroskopischer Ebene entsteht somit eine Störung der Darmintegrität, was Toxinen und Antigenen erlaubt, in Kontakt mit dem Immunsystem zu kommen und Abwehrreaktionen auszulösen. Dies führt zur Freisetzung von Komplementfaktoren, TNF-alpha und Interferon-gamma und damit zu einer Inflammation und weiteren Störung der Integrität. So kann eine einmalige Störung der Schutzfunktionen der Darmwand eine Entzündungsreaktion initiieren, die dann unabhängig vom auslösenden Agens weiter bestehen bleibt [6].

\section{Enterales Nervensystem}

Das enterale Nervensystem steuert sowohl die Motorik des Darms als auch komplizierte Stoffwechselfunktionen wie die Sekretion von Elektrolyten oder die Nahrungsaufnahme. In den vergangenen Jahren konnten im enteralen Nervensystem sämtliche Neurotransmitter gefunden werden, die aus dem zentralen Nervensystem bekannt sind. Insgesamt verfügt das enterale Nervensystem über 100000 Neuronen, weshalb es zuweilen als Bauchhirn bezeichnet wird [7]. Dieses Nervensystem wird dem Unbewussten zugeordnet, weist eine ausgesprochene Neuroplastizität auf und interagiert sehr eng mit dem mukosalen Immunsystem [8].

Interessant in diesem Zusammenhang ist die Tatsache, dass der Nervus vagus im Gegensatz zu früheren Ansichten eine bidirektionale Funktion innehat: Ein afferenter Anteil des Informationsflusses gelangt so aus dem enteralen in das zentrale Nervensystem $[9,10]$. Die Darmflora beeinflusst auf diese Weise über das enterische Nervensystem und den N. vagus unsere Emotionen und unser Verhalten
[11]. Ausdrücke wie das «Bauchgefühl» oder «aus dem Bauch heraus entscheiden» finden somit eine neue Erklärungsgrundlage. Genau wie im zentralen Nervensystem zeigt auch das enterale Nervensystem eine Neuroplastizität in Bezug auf das Schmerzgedächtnis, was ein neues Licht auf die Pathogenese des Reizdarmsyndroms wirft [12].

Die Entdeckung des extraneuronalen cholinergen Systems hat zu einer Erweiterung unseres Verständnisses sowohl der Psychosomatik als auch der Somatopsychik geführt. Hier werden cholinerge parasympathische Nervenimpulse über ein System aus Fibroblasten und Immunzellen geleitet. Auf dieser Ebene sind also das Vegetativum und das Immunsystem funktionell vereint [13]. Diese Erkenntnisse werden in Zukunft einen integrativen Ansatz in der Medizin notwendig machen.

\section{Pathogenese der chronisch- entzündlichen Darmerkrankungen aus ganzheitlicher Sicht}

Die Veränderung der natürlichen intestinalen Darmflora spielt eine Schlüsselrolle in der Pathogenese von CED. Man geht davon aus, dass durch den Verlust der Toleranz gegenüber eigenen Darmbakterien die lokalen Entzündungsprozesse im gastrointestinalen Immunsystem ausgelöst werden [14]. Die gegen veränderte Darmflora gerichtete Immunantwort führt allmählich $\mathrm{zu}$ einer strukturellen Schädigung der Schleimhaut und somit auch zur Zerstörung der natürlichen Darmbarriere. Eine anhaltende Stimulierung der Immunprozesse hat eine systemische Auswirkung mit Befall extraintestinaler Organe, wie z.B. Gelenke oder Haut, zur Folge. Obwohl Morbus Crohn das klassische Beispiel für die systemische Ausprägung einer chronischen Darmentzündung darstellt, treten ähnliche Reaktionen ebenfalls bei Colitis ulcerosa auf.
In Anbetracht dieser pathogenetischen Kette soll eine ganzheitliche Betrachtung und Behandlung von CED mindestens die beiden oben dargestellten Aspekte - Darmflora und Immunsystem - mitberücksichtigen. Darüber hinaus dürfen in der eingehenden Analyse von CED die psychologischen und sozialen Aspekte nicht fehlen. Colitis ulcerosa und Morbus Crohn werden seit Jahrzehnten als klassische Beispiele für psychosomatische Erkrankungen mit einem charakteristischen Persönlichkeitsmuster der Betroffenen angeführt. Vor diesem Hintergrund stehen für eine holistische Behandlung der CED folgende Ansätze: Darmflora, Immunsystem und Psyche. Um all diesen Aspekten Rechnung zu tragen, sollen die standardmässigen Methoden der konventionellen Medizin mit den bewährten Massnahmen der Komplementärmedizin im Sinne eines integrativen Ansatzes kombiniert werden. Aufgrund der Komplexität und Schwere der Krankheitsverläufe dürfen die komplementärmedizinischen Methoden kaum als Alternative zur Schulmedizin, sondern ergänzend und supportiv (komplementäre Medizin) eingesetzt werden.

\section{Möglichkeiten der ganzheitlichen Abklärung}

Mit den modernen, vor allem endoskopischen Untersuchungsmethoden der Gastroenterologie ist ein präziser Nachweis struktureller Veränderungen im gesamten Gastrointestinaltrakt gegeben. Die seit Neuem vorhandene Calprotectin-Bestimmung im Stuhl erlaubt eine gezielte Beurteilung des lokalen Entzündungsgrades. Für die funktionellen Aspekte der CED bietet die Komplementärmedizin weitere interessante und bewährte Tests. Einer der wichtigsten ist die Stuhlflora-Analyse. Diese mikrobiologische Untersuchungsmethode wurde in den 1950er-Jahren entwickelt und im Laufe der Zeit verfeinert 
und standardisiert. Dabei werden die bekannten Schlüsselbakterien - aufgeteilt auf Protektivflora, immunomodulierende und proteolytische Flora - quantitativ bestimmt. Die Stuhlflora-Analyse bietet sowohl relevante Informationen über den $\mathrm{Zu}$ stand der Mikroökologie des Darms als auch Hinweise auf die mögliche probiotische Behandlung. Nach den gesammelten Erfahrungen in der Aeskulap-Klinik weisen viele CEDPatienten einen Mangel an Protektivflora (Bifidobakterien und Laktobazillen) auf. Als Folge kommt es oft $\mathrm{zu}$ einem übermässigen Wachstum von proteolytischer Flora, insbesondere der Clostridien. Eine schwere Floradysbalance führt sekundär zum Wachstum von Pilzen, z.B. Candida oder Befall mit Blastocystis hominis und Helicobacter-Spezies.

Wertvolle Aufschlüsse über die Störung der Ausscheidungsfunktion des Verdauungstrakts liefert der einfache Uricolor-Test im Urin. Durch die farbige biochemische Reaktion des Harns nach Überschichtung mit Salpetersäure wird die übermässige Menge an Indikan und Urobilinogen dargestellt.

Eine vermehrte Permeabilität des Darms wird durch die Bestimmung von Alpha-1-Antitrypsin im Stuhl beurteilt. Eine Störung der Darmbarriere verursacht eine pathologische Antigenbelastung vonseiten konsumierter Lebensmittel. Seit einigen Jahren ist es möglich, die immunologische Antwort unseres Organismus auf gängige Lebensmittel mittels Bestimmung von IgG, im Besonderen $\operatorname{IgG} 4$, zu evaluieren. Es ist wichtig festzuhalten, dass es sich bei diesem immunologischen Test um keine Allergietestung handelt, sondern um eine Beurteilung der pathologischen Belastung infolge einer verstärkten Permeabilität des Darms. Ein Einfluss der IgG-Antikörper auf die Entstehung diverser Krankheiten, z.B. der Migräne im Sinne der subklinischen Entzündung (low-grade inflammation), wurde beschrieben [15]. Aufgrund der Ergebnisse ist es möglich, den Patienten durch eine gezielte Eliminationsdiät vorübergehend immunologisch zu entlasten. Diese einfache Massnahme führt gemäss unserer Erfahrung bei vielen Patienten $\mathrm{zu}$ einer wesentlichen Linderung initialer Beschwerden. Sobald die Integrität der Schleimhautbarriere hergestellt ist, dürfen alle Lebensmittel allmählich wieder konsumiert werden [16].

In Bezug auf die Ernährung von CED-Patienten wird aus ganzheitlicher Sicht gezielt die Problematik einer Histamin-Intoleranz evaluiert. Diverse Bioamine, insbesondere Histamin, entstehen bei fermentativer Herstellung und Konservierung bestimmter Lebensmittel, wie z.B. Wein, Käse oder Sauerkraut. Falls Histamin aus den Lebensmitteln nicht genügend durch die enterale Diaminooxidase (DAO) abgebaut wird, kommt es $\mathrm{zu}$ den typischen Beschwerden wie Kopfschmerzen, Atembeschwerden, Hautausschlägen oder Blähungen, Diarrhö und Bauchkoliken. Obwohl die DAO-Aktivität im Blut nicht immer mit der Ausprägung der Krankheit korreliert, bietet sie einen wertvollen Hinweis für eine weitere Beurteilung und diätetische Behandlung dieser Störung [17].

\section{Literatur}

1 Jacobsen BA, Fallingborg J, Rasmussen $\mathrm{HH}$, et al: Increase in incidence and prevalence of inflammatory bowel disease in northern Denmark: a population-based study, 1978-2002. Europ J Gastroenter Hepatol 2006;18:601-606

2 Rogler G, Scharl M: Inflammatory bowel disease pathogenesis: what is new? Curr Opin Gastroenterol 2012;28:301-309.

3 Microbiome Online. https://en.wikipedia. org/wiki/Microbiome.

4 Ivanov II, Frutos Rde L, Manel N, et al: Specific microbiota direct the differentiation of Th17 cells in the mucosa of the small intestine. Cell Host Microbe 2008;4:337-349.

5 Hollander D: Intestinal permeability, leaky gut and intestinal disorders. Curr Gastroenterol Rep 1999;5:410-416.

6 Fasano A: Leaky gut and autoimmune diseases. Clin Rev Allergy Immunol 2012;42: 71-78.

7 Gershon MD: The enteric nervous system: a second brain. Hosp Pract (1995) 1999; 34:31-32, 35-38, 41-42.

8 Gershon MD: Nerves, reflexes and the enteric nervous system: pathogenesis of the irritable bowel syndrome. J Clin Gastroenterol 2005;39(suppl 3):184-193.

9 Ochoa-Repáraz J, Mielcarz DW, BegumHaque S, Kasper LH: Gut, bugs and brain: role of commensal bacteria in the control of central nervous system disease. Ann Neurol 2011;69:240-247.

10 Forsythe P, Kunze WA: Voices from within: gut microbes and the CNS. Cell Mol Life Sci 2013;70:55-69.

11 Messaoudi M, Lalonde R, Violle $\mathrm{N}$, et al: Assessment of psychotropic-like properties of a probiotic formulation. Br J Nutr 2011;105:755-764.

12 Layer P, Andresen V, Pehl C, et al: S3Leitlinie Reizdarmsyndrom: Definition, Pathophysiologie, Diagnostik und Therapie. Z Gastroenterol 2011;49:237-293.

13 Kawashima K, Fujii T: Extraneuronal cholinergic system in lymphocytes. Pharmacol Ther 2000;86:29-48.

14 Sartor RB: Intestinal microflora in human and experimental inflammatory bowel disease. Curr Opin Gastroenterol 2001;17:324330.

15 Arroyave Hernández CM, Echavarría Pinto M, Hernández Montiel HL: Food allergy mediated by IgG antibodies associated with migraine in adults. Rev Alerg Mex 2007;54:162-168.

16 Gabele H: Nahrungsmittelunverträglichkeit ganzheitlich betrachtet. Schweiz Z Ganzheitsmed 2011;23:88-93.

17 Maintz L, Novak N: Histamine and histamine intolerance. Am J Clin Nutr 2007;85: 1185-1196. 